\title{
Utilização e acesso de idosos a serviços de atenção básica em Porto Alegre (RS, Brasil)
}

\author{
Use and access of the elderly to primary health care services \\ in Porto Alegre (RS, Brasil)
}

Lisiane Manganelli Girardi Paskulin ${ }^{1}$

Daiany Borghetti Valer ${ }^{1}$

Lucila Amaral Carneiro Vianna ${ }^{2}$

${ }^{1}$ Departamento de Assistência e Orientação Profissional, Escola de Enfermagem, Universidade Federal do Rio Grande do Sul. Rua São Manoel 963, Rio Branco. 90620-110 Porto Alegre RS. paskulin@orion.ufrgs.br ${ }^{2}$ Departamento de Enfermagem, Universidade Federal de São Paulo.
Abstract A cross-sectional study was carried out in the second semester of 2004 by means of a household survey with 292 elderly people in a Southern Brazilian city, selected by probabilistic sampling. The objectives were: to describe the use and geographic access of the elderly to primary healthcare (PHC) in Porto Alegre (RS), and to analyze the association between variables of interest to the study and access to PHC. Descriptive and bivariate analysis was used. The primary health care services were used by $49.7 \%$ of the respondents, motivated by their location, quality and "free service." The factors negatively associated with the use of PHC services were "level of education" and "perception of good health" and the variable related positively was "self-diagnosed chronic condition". The elderly exposed to frailty related with economic and health conditions were more likely to use PHC, showing signs of equity in the health system. On the other hand, it is necessary to broaden the coverage to the elderly that do not have access, as well as implement healthcare strategies to meet the heterogenous demands of this age group.

Key words Access to Healthcare, Elderly, Health of the elderly, Health services, Primary Health Care, Population ageing
Resumo Trata-se de um estudo transversal realizado no segundo semestre de 2004 por meio de inquérito domiciliar com 292 idosos do distrito Noroeste de Porto Alegre (RS), selecionados por amostra probabilística. Teve por objetivos: descrever a utilização e acesso geográfico de idosos a serviços de atenção básica (SAB) e analisar a associação entre variáveis de interesse do estudo e a utilização dos SAB. Realizou-se análise descritiva e bivariável. Os resultados mostraram que os $S A B$ foram utilizados por $49,7 \%$ dos idosos, motivados por sua localização, qualidade e "gratuida$d e$ ". Os fatores associados negativamente à utilização do SAB foram "escolaridade" e o fato de "perceber-se saudável", e a variável associada positivamente foi "autorrelato de dano crônico". Idosos expostos a situações de fragilidade relacionadas às condições econômicas e de saúde foram os que mais utilizaram um $S A B$, demonstrando sinais de equidade do sistema. No entanto, é necessário ampliar a cobertura àqueles que não conseguem acesso, bem como as estratégias de atenção para dar conta da heterogeneidade de demandas desse grupo etário.

Palavras-chave Acesso aos serviços de saúde, Idoso, Saúde do idoso, Serviços de saúde, Atenção Primária (Básica) à Saúde, Envelhecimento populacional 


\section{Introdução}

A mudança do perfil demográfico no cenário mundial e, de forma mais recente, no Brasil tem, entre suas manifestações, o aumento no número de idosos. Esse fenômeno traz consigo grande impacto social, exigindo mudanças desde o nível das políticas públicas até o do núcleo familiar. Na perspectiva do envelhecimento populacional, é comprovado que os idosos são grandes usuários dos serviços de saúde ${ }^{1,2}$ e que vem ocorrendo um aumento no consumo dos serviços por parte desse grupo populacional nos últimos anos ${ }^{3}$.

O uso dos serviços de saúde representa todo o contato direto ou indireto que os usuários têm com eles ${ }^{4}$. Avaliar a sua utilização é um modo de compreender também o seu acesso ${ }^{2}$. Conforme Unglert ${ }^{5}, \mathrm{o}$ acesso aos serviços pode ser conceituado como a disponibilidade dos serviços de saúde e sua adequada cobertura, e está diretamente relacionado ao processo saúde-doença. Ele envolve aspectos geográficos, funcionais, socioculturais e econômicos ${ }^{5,6}$.

Estudos brasileiros de natureza empírica têm apontado fatores que influenciam a utilização dos serviços de saúde tanto por parte da população em geral quanto pelo idoso, tais como oferta, características demográficas, geográficas, econômicas e sociais, além das necessidades de saú$\mathrm{de}^{2,7-10}$. Travassos e Martins ${ }^{4}$ afirmam que a influência desses fatores varia em razão do tipo de serviço e da proposta assistencial.

Associado ao acesso, encontra-se ainda o princípio de resolutividade, que diz respeito à capacidade do sistema em resolver situações relacionadas à saúde/doença dos usuários e/ou atendê-los de forma adequada em todos os níveis de atenção. Engloba desde aspectos relativos à percepção dos usuários, passando por questões relacionadas à estruturação dos serviços, até a organização do sistema de saúde ${ }^{11}$.

Visando contribuir para o planejamento em saúde local de uma das regiões com maior proporção de idosos do município de Porto Alegre, Rio Grande do Sul, as questões que nortearam este estudo foram: que motivos levam esse grupo populacional a utilizar (ou não) um serviço de atenção básica (SAB)? Como se deslocam ao $\mathrm{SAB}$ ? Em quanto tempo? Com que frequência e finalidade procuram um SAB? Consideram o $S A B$ próximo de suas residências? Percebem ter suas demandas atendidas? Quais os fatores associados à utilização de um SAB?

A opção por investigar aspectos relacionados ao acesso e à utilização dos $\mathrm{SAB}$ parte do princí- pio de que esses serviços deveriam ser a porta de entrada do sistema de saúde, assim como resolver em torno de $80 \%$ das necessidades de saúde da sua população adscrita ${ }^{12}$. Verifica-se ainda que poucos estudos têm enfocado, de modo mais específico, a utilização dos $S A B$ por idosos ${ }^{13,14}$. Para responder às questões levantadas anteriormente, foram estabelecidos os seguintes objetivos: descrever a utilização e acesso geográfico de idosos a serviços de atenção básica e analisar a associação entre variáveis de interesse do estudo e a utilização dos SAB.

\section{Métodos}

Trata-se de um estudo epidemiológico observacional e transversal ${ }^{15}$, realizado por meio de inquérito domiciliar com pessoas de 60 anos ou mais, moradoras do distrito Noroeste de Porto Alegre, no segundo semestre de 2004. A região Noroeste é um dos 16 distritos sanitários da cidade. Conta com os seguintes serviços de atenção básica: um centro de saúde, cinco unidades básicas de saúde, uma unidade da Estratégia de Saúde da Família (ESF), além de ambulatórios especializados e hospitais. Pela política de saúde municipal, esses SAB possuem áreas de abrangência definidas e os usuários são atendidos de acordo com seu local de residência.

O número de indivíduos que fizeram parte da amostra foi estabelecido por meio de cálculo estatístico baseado no número das variáveis de interesse do estudo ${ }^{16}$, prevendo $5 \%$ de perdas por problemas de preenchimento dos instrumentos de pesquisa. O poder de estudo adotado foi de 90\%, com nível de significância de 0,05 e tamanho de efeito de 0,6 . Assim, a estimativa do tamanho da amostra era de 294 indivíduos. Para alcançar o número proposto de indivíduos, foi realizada uma amostragem probabilística em dois estágios. No primeiro, foram sorteadas as ruas do distrito de maneira sistemática; no segundo, sortearam-se os domicílios por numeração, considerando-se o início e fim da rua e lados par e ímpar. Em cada uma das ruas foram identificados três idosos que preenchiam os seguintes critérios de inclusão: ter 60 anos ou mais, ter condições de responder ao instrumento de pesquisa, ser morador da área de abrangência do respectivo distrito e aceitar participar do estudo. Os indivíduos sem condições de responder ao instrumento foram excluídos por questões metodológicas do estudo, pois foi proposto investigar a percepção dos sujeitos. As entrevistas fo- 
ram realizadas no segundo semestre de 2004 pela pesquisadora e por pessoal treinado sob sua supervisão a fim de parear os indivíduos que fariam parte da coleta dos dados.

Em relação à utilização dos $\mathrm{SAB}$, foram coletadas as seguintes informações: tipo de serviço de saúde utilizado nos seis meses anteriores ao inquérito, motivos para ter utilizado ou não um SAB, percepção de proximidade do SAB utilizado, modo e tempo de deslocamento, percepção de resolutividade, finalidade e frequência de uso do SAB.

Para a análise de associação, foi considerada como variável de desfecho a utilização (ou não) de um SAB nos seis meses anteriores à entrevista. Foram considerados como SAB as unidades de saúde, centro de saúde e unidades da ESF. As variáveis de interesse foram: demográficas (sexo e grupo etário), socioeconômicas (estado conjugal, escolaridade, arranjo domiciliar e percepção de contar com rede de apoio social informal), relacionadas à saúde (condição subjetiva de saúde e autorrelato de dano crônico), além da capacidade funcional. A estratificação das faixas etárias foi: 60 a 69 anos, 70 a 79 anos e 80 anos ou mais. A capacidade funcional foi avaliada pelo grau de dependência do idoso nas Atividades da Vida Diária (AVD) segundo categorias estabelecidas por Ramos et al. ${ }^{17}$ : independentes (não necessitavam de ajuda para realizar nenhuma AVD), dependentes leves (necessitavam de ajuda para uma a três AVD), dependentes moderados (necessitavam de ajuda para realizar quatro a seis AVD) e dependentes severos (necessitavam de ajuda para sete ou mais AVD).

Dos 385 idosos contatados, foram excluídos do estudo 15 idosos por não terem condições de responder às questões da entrevista, e outros 76 não aceitaram participar do estudo (taxa de recusa de 19,8\%). Além disso, dois sujeitos foram excluídos por não serem residentes na área de abrangência. Portanto, participaram do estudo 292 idosos, sendo a estimativa de amostra de 294 indivíduos. Os dados foram gerenciados pelo programa SPSS 12.0 e foram realizadas análise descritiva (frequência absoluta e relativa) e o teste qui-quadrado de Pearson (nível de significância de 0,05$)$.

O projeto de pesquisa foi aprovado pelo Comitê de Ética em Pesquisa da Universidade Federal de São Paulo. Os participantes assinaram termo de consentimento informado.

\section{Resultados}

Os SAB foram utilizados por 145 (49,7\%) idosos durante os seis meses anteriores à entrevista e foram os serviços mais citados $(34,8 \%)$, conforme a Tabela 1. O principal motivo referido pelos respondentes para não terem utilizado um SAB foi o fato de terem plano de saúde (46,9\%). Entre os motivos relatados para utilização dos SAB, destacam-se: localização próxima à residência (28,5\%); considerarem um bom serviço $(26,5 \%)$ e a gratuidade $(22,9 \%)$.

Em relação à percepção do idoso sobre a proximidade do SAB (Tabela 2), dos 143 idosos que afirmaram ter utilizado o mesmo, a maioria $(91,6 \%)$ considerou a unidade próxima à sua residência. Os idosos deslocavam-se até o SAB es-

Tabela 1. Serviços de saúde utilizados e motivos de utilização e não utilização de um SAB para idosos do Distrito Noroeste Porto Alegre (RS), 2004.

\begin{tabular}{lrr}
\hline \multicolumn{1}{c}{ Utilização dos serviços e motivos } & Total & $\mathbf{f}(\%)$ \\
\hline Tipo de serviço utilizado & & \\
Serviço público de atenção básica & 145 & 34,8 \\
Clínica/ambulatório com plano de saúde & 107 & 25,7 \\
Não utilizou & 41 & 9,8 \\
Clínica/ambulatório público & 38 & 9,1 \\
Emergência pública & 22 & 5,3 \\
Hospital com plano de saúde & 16 & 3,8 \\
Clínica/ ambulatório com pagamento direto & 15 & 3,6 \\
Hospital público & 13 & 3,1 \\
Emergência com plano de saúde & 12 & 2,9 \\
Outros & 06 & 1,4 \\
Hospital com pagamento direto & 02 & 0,5 \\
Total (N=292) * & 417 & 100,0 \\
Motivo para não utilizar um SAB & & \\
Tem plano de saúde & 90 & 46,9 \\
Não precisou & 36 & 18,8 \\
Tem problemas de acesso & 32 & 16,4 \\
Prefere atendimento da rede privada & 14 & 7,3 \\
Outros & 20 & 10,4 \\
Total (N=147) ${ }^{* *}$ & 192 & 100,0 \\
Motivo para utilizar um SAB & & \\
Pela localização & 72 & 28,5 \\
Gosta, considera um bom serviço & 67 & 26,5 \\
Não precisa pagar & 58 & 22,9 \\
Outros & 41 & 16,3 \\
Para alguma atividade específica: vacina & 15 & 5,9 \\
Total (N=145) ${ }^{*}$ & 253 & 100,0 \\
& & \\
& & \\
& & \\
& &
\end{tabular}

Notas: * O número de serviços utilizados foi maior que o número de idosos porque alguns utilizaram mais de um serviço no período; ${ }^{* *} \mathrm{O}$ número total de motivos foi superior ao número de respondentes que utilizaram ou não um SAB porque alguns idosos apresentaram mais de um motivo. 
sencialmente a pé $(45,6 \%)$ e de ônibus $(29,1 \%)$. A maior parte $(59,6 \%)$ relatou levar até dez minutos para o deslocamento.

Para 76,4\% dos idosos que utilizavam um $\mathrm{SAB}$, seus problemas eram resolvidos. A vacina-

Tabela 2. Utilização do SAB, acesso e resolutividade para idosos do distrito Noroeste - Porto Alegre (RS), 2004.

\begin{tabular}{|c|c|c|}
\hline $\begin{array}{l}\text { Utilização, acesso ao } S A B \\
\text { e resolutividade }\end{array}$ & Total & $\mathbf{f}(\%)$ \\
\hline \multicolumn{3}{|l|}{ Percepção proximidade } \\
\hline Sim & 131 & 91,6 \\
\hline Não & 10 & 7,0 \\
\hline Regular & 2 & 1,4 \\
\hline Total * & 143 & 100,0 \\
\hline \multicolumn{3}{|l|}{ Modo de deslocamento } \\
\hline A pé & 72 & 45,6 \\
\hline Ônibus & 46 & 29,1 \\
\hline Carro & 25 & 15,8 \\
\hline Táxi & 7 & 4,4 \\
\hline Outros & 8 & 5,1 \\
\hline Total ${ }^{* *}$ & 158 & 100,0 \\
\hline \multicolumn{3}{|l|}{ Tempo de deslocamento } \\
\hline Até 10 minutos & 87 & 59,6 \\
\hline 11 a 20 minutos & 38 & 26,0 \\
\hline 21 ou mais minutos & 19 & 13,0 \\
\hline Não sabe & 2 & 1,4 \\
\hline Total ${ }^{* *}$ & 146 & 100,0 \\
\hline \multicolumn{3}{|l|}{ Finalidade de uso } \\
\hline Vacinas & 95 & 24,0 \\
\hline Consulta médica & 94 & 23,7 \\
\hline Buscar medicação & 77 & 19,4 \\
\hline Procedimentos de enfermagem & 69 & 17,4 \\
\hline Fazer exames & 42 & 10,6 \\
\hline Consulta com outros profissionais & 10 & 2,6 \\
\hline Participar de grupos & 5 & 1,3 \\
\hline Outros & 4 & 1,0 \\
\hline Total ${ }^{* *}$ & 396 & 100,0 \\
\hline \multicolumn{3}{|l|}{ Frequência de uso } \\
\hline Uma vez nos últimos seis meses & 39 & 27,1 \\
\hline Três vezes nos últimos seis meses & 39 & 27,1 \\
\hline Uma vez ao mês & 30 & 20,8 \\
\hline Duas vezes nos últimos seis meses & 21 & 14,6 \\
\hline Mais de uma vez ao mês & 12 & 8,3 \\
\hline Não lembra & 3 & 2,1 \\
\hline Total ${ }^{* * *}$ & 144 & 100,0 \\
\hline \multicolumn{3}{|l|}{ Percepção de resolutividade } \\
\hline $\operatorname{Sim}$ & 110 & 76,4 \\
\hline Às vezes & 29 & 3,5 \\
\hline Não & 5 & 20,1 \\
\hline Total ${ }^{* * *}$ & 144 & 100,0 \\
\hline
\end{tabular}

Notas: " Dois idosos não responderam; "* Alguns idosos apresentaram mais de uma resposta; ${ }^{* *+}$ Um idoso não respondeu. ção $(24,0 \%)$ e a consulta médica $(23,7 \%)$ foram as principais finalidades de uso. Quanto à frequência de utilização dos serviços, houve distribuição semelhante entre aqueles idosos que utilizaram o SAB de uma a três vezes $(27,1 \%)$ nos seis meses anteriores à entrevista.

Em relação às características socioeconômicas e demográficas (Tabela 3), verificou-se que a maioria dos respondentes era do sexo feminino $(67,8 \%)$ e que houve distribuição semelhante entre respondentes nas faixas de 70 a 79 anos $(42,8 \%)$ e de 60 a 69 anos $(42,5 \%)$. Observou-se ainda que não houve associação significativa entre a utilização de um SAB e as variáveis sexo $(p=0,561)$ e idade $(p=0,696)$. Dos respondentes, $53,1 \%$ viviam sem companheiro, e essa variável também não se mostrou associada $(p=0,238)$ à utilização de um SAB.

Com relação à escolaridade, houve maior proporção de idosos $(43,6 \%)$ com os primeiros quatro anos completos (antigo primário). Houve associação significativa entre escolaridade e utilização de um SAB $(p<0,001)$, na qual a maioria dos idosos analfabetos ou com primário incompleto $(72,1 \%)$ utilizava um SAB, e os que possuíam ensino médio e superior não o utiliza$\operatorname{vam}(74,0 \%)$.

No que se refere ao arranjo domiciliar, a maioria dos idosos residia acompanhado $(78,1 \%)$ e a variável não apresentou associação significativa $(p=0,227)$ com utilização dos SAB. Verificouse que pouco menos da metade dos entrevistados $(49,5 \%)$ relatou perceber estar recebendo apoio da rede social informal. Essa variável também não mostrou associação significativa $(p=0,598)$ com a utilização dos SAB.

Observou-se ainda que a maioria $(81,0 \%)$ se considerou saudável e houve associação significativa entre condição subjetiva de saúde e utilização de um SAB $(p=0,008)$. Os idosos que se consideravam saudáveis não utilizaram um SAB $(54,3 \%)$, e entre aqueles que se consideravam doentes, a maioria utilizou SAB (65,5\%).

Referiram ser portadores de dano crônico $69,2 \%$ dos idosos, sendo que também houve associação entre essa variável e a utilização do SAB $(p=0,007)$. A maior parte dos idosos que relatavam ser portadores de dano crônico utilizava $S A B$ $(55,0 \%)$, e entre os que não relatavam, a maior parte não fazia uso de $\operatorname{SAB}(62,2 \%)$.

Quanto às AVD, a maioria $(63,7 \%)$ dos participantes do estudo se mostrou independente na realização das mesmas, mas essa variável não mostrou associação significativa com a utilização de um SAB $(p=0,257)$. 
Tabela 3. Características socioeconômicas, demográficas e de saúde dos idosos do Distrito Sanitário Noroeste de Porto Alegre segundo utilização dos SAB - Porto Alegre (RS), 2004.

\begin{tabular}{|c|c|c|c|c|c|}
\hline Características & Total & $f(\%)$ & $\begin{array}{c}\text { Utiliza serviço de } \\
\text { atenção básica - f (\%) }\end{array}$ & $\begin{array}{l}\text { Não utiliza serviço de } \\
\text { atenção básica - f (\%) }\end{array}$ & $p$ \\
\hline Sexo & & & & & 0,561 \\
\hline Feminino & 198 & $(67,8)$ & $96(48,5)$ & $102(51,5)$ & \\
\hline Masculino & 94 & $(32,2)$ & $49(52,1)$ & $45(47,9)$ & \\
\hline Total & 292 & $(100,0)$ & - & - & 0,696 \\
\hline \multicolumn{6}{|l|}{ Grupo etário } \\
\hline $70 \vdash 79$ anos & 125 & $(42,8)$ & $65(52,0)$ & $60(48,0)$ & \\
\hline $60 \vdash 69$ anos & 124 & $(42,5)$ & $58(46,8)$ & $66(53,2)$ & \\
\hline 80 anos ou mais & 43 & $(14,7)$ & $22(51,2)$ & $21(48,8)$ & \\
\hline Total & 292 & $(100,0)$ & - & - & 0,238 \\
\hline \multicolumn{6}{|l|}{ Estado conjugal } \\
\hline Sem companheiro & 155 & $(53,1)$ & $82(52,9)$ & $73(47,1)$ & \\
\hline Com companheiro & 137 & $(46,9)$ & $63(46,0)$ & $74(54,0)$ & \\
\hline Total & 292 & $(100,0)$ & - & - & 0,000 \\
\hline \multicolumn{6}{|l|}{ Escolaridade } \\
\hline Primário completo/ginásio & 127 & $(43,6)$ & $70(55,1)$ & $57(44,9)$ & \\
\hline Analfabeto/primário incompleto & 68 & $(23,4)$ & $49(72,1)$ & $19(27,9)$ & \\
\hline Ensino médio/superior & 96 & $(33,0)$ & $25(26,0)$ & $71(74,0)$ & \\
\hline Total $^{*}$ & 291 & $(100,0)$ & - & - & 0,227 \\
\hline \multicolumn{6}{|l|}{ Arranjo domiciliar } \\
\hline Outras configurações & 151 & $(51,7)$ & $73(48,3)$ & $78(51,7)$ & \\
\hline Domicílio unigeracional & 77 & $(26,4)$ & $39(50,6)$ & $38(49,4)$ & \\
\hline Reside só & 64 & $(21,9)$ & $33(51,6)$ & $31(48,4)$ & \\
\hline Total & 292 & $(100,0)$ & - & - & 0,598 \\
\hline \multicolumn{6}{|l|}{ Apoio social informal } \\
\hline Não & 147 & $(50,5)$ & $71(48,3)$ & $76(51,7)$ & \\
\hline Sim & 144 & $(49,5)$ & $74(51,4)$ & $70(48,6)$ & \\
\hline Total * & 291 & $(100,0)$ & - & - & 0,008 \\
\hline \multicolumn{6}{|l|}{ Condição de saúde } \\
\hline Saudável & 234 & $(81,0)$ & $107(45,7)$ & $127(54,3)$ & \\
\hline Doente & 55 & $(19,0)$ & $36(65,5)$ & $19(34,5)$ & \\
\hline Total * & 289 & $(100,0)$ & - & - & 0,007 \\
\hline \multicolumn{6}{|l|}{ Autorrelato de dano crônico } \\
\hline Sim & 202 & $(69,2)$ & $111(55,0)$ & $91(45,0)$ & \\
\hline Não & 90 & $(30,8)$ & $34(37,8)$ & $56(62,2)$ & \\
\hline Total & 292 & $(100,0)$ & - & - & 0,257 \\
\hline \multicolumn{6}{|l|}{ Atividades da vida diária } \\
\hline Independente & 183 & $(63,7)$ & $91(49,7)$ & $92(50,3)$ & \\
\hline Dependente leve & 70 & $(24,4)$ & $30(42,9)$ & $40(57,1)$ & \\
\hline Dependente moderado & 20 & $(7,0)$ & $9(45,0)$ & $11(55,0)$ & \\
\hline Dependente severo & 14 & $(4,9)$ & $10(71,4)$ & $4(28,6)$ & \\
\hline Total * & 287 & $(100,0)$ & - & - & \\
\hline
\end{tabular}

Nota: ${ }^{*}$ Um ou mais idosos não responderam.

\section{Discussão}

Estudos transversais apresentam limitações no tocante à identificação temporal dos fatores estudados, mas o presente estudo permitiu obter um panorama sobre aspectos relacionados à utilização dos SAB e percepção de idosos sobre seu acesso geográfico a esses serviços, constituindo dados primários, coletados para esse fim. No entanto, apesar dos esforços para obtenção de uma amostra probabilística, não foi possível determinar quão similares ou diferentes eram outros idosos moradores da região que não participaram do estudo. A pesquisa permitiu não só 
analisar aspectos relacionados ao acesso e utilização dos SAB na perspectiva dos usuários desses serviços, mas também analisar os motivos que levaram idosos da comunidade a não utilizarem esse tipo de serviço. Verificou-se ainda que a taxa de recusa em participar da investigação foi elevada, o que deve estar relacionado à situação de insegurança vivenciada pelos brasileiros, fato observado durante a coleta de dados. Entretanto, a recusa em participar ocorreu de forma relativamente homogênea nas ruas sorteadas.

Outra limitação foi a exclusão de idosos que não tiveram condições de responder à entrevista em razão de questões metodológicas da pesquisa, que objetivavam conhecer aspectos referentes à percepção, critério já utilizado em outros estudos epidemiológicos ${ }^{14,18}$.

Considerando-se todos os serviços utilizados, o SAB foi o serviço mais citado entre os respondentes. Porém, é importante notar que o número total de serviços citados foi superior ao número de idosos, o que pode ter implicado no uso de mais de uma modalidade de serviços públicos ou privados simultaneamente ou de mais de um nível de atenção. Segundo a Pesquisa Nacional de Amostragem por Domicílios (PNAD) de $2003^{19}$, os postos ou centros de saúde foram os serviços utilizados majoritariamente pela população em geral nos 12 meses anteriores à entrevista, numa proporção de 52,5\%. Traçando aproximações entre os dados da PNAD e os resultados do presente estudo, poder-se-ia dizer que as proporções de utilização dos serviços públicos de atenção básica são semelhantes, apesar de ser considerado um período de tempo diferente e faixas etárias diferentes.

Verificou-se ainda que a utilização de serviços privados com pagamento direto foi bastante restrita. Após os serviços públicos, o uso de serviços privados com plano de saúde foi o mais freqüente. Somando-se todos os valores referentes à utilização de serviços, os serviços públicos foram citados 218 vezes enquanto que os privados com pagamento direto ou com plano de saúde o foram 152 vezes, correspondendo a 58,9\% e $41,1 \%$, respectivamente. Bós e Bós ${ }^{7}$ identificaram que $55,8 \%$ da população idosa gaúcha utilizaram o sistema público de saúde, e Venturi et al. ${ }^{20}$ encontraram um percentual de 45,2\%, em Viçosa (MG). No presente estudo, entretanto, não se pode afirmar que os idosos que fizeram uso dos serviços públicos também não utilizaram um serviço privado ou vice-versa.

Em relação às respostas referentes aos motivos para a não utilização do $S A B$, destaca-se que
90 idosos (30,8\% dos 292) responderam possuir cobertura de um plano de saúde. Esses valores se aproximam dos dados divulgados pela PNAD de $2003^{19}$, nos quais $29,8 \%$ da população brasileira com 65 anos ou mais estava coberta por algum tipo de plano de saúde. Durante as entrevistas, os idosos referiram que se sentiam mais seguros quanto ao acesso rápido para atendimento ambulatorial e hospitalar por possuírem um plano de saúde. Entretanto, acredita-se que, de modo geral, as ações disponibilizadas pelos serviços complementares são predominantemente de caráter curativo e individual. Assim, mesmo que os idosos tenham acesso facilitado por disporem de um plano de saúde, ações de promoção da saúde e de prevenção de doenças, e mesmo de atendimento domiciliário, ainda necessitam ser ampliadas ou implementadas nos serviços suplementares para atenderem aos princípios da Política Nacional de Saúde da Pessoa Idosa ${ }^{21}$.

Observa-se que alguns idosos perceberam dificuldade de acesso, demonstrando que os serviços de atenção básica não têm funcionado como porta de entrada para todos os idosos que deles dependem. A ampliação do acesso seria um importante fator para redução das iniqüidades em saúde ${ }^{22}$. Apesar de não ter sido objeto de investigação nesta pesquisa, salienta-se que outros estudos ${ }^{9,23}$ já demonstraram que os usuários com mais baixo nível socioeconômico são os que têm maior dificuldade em conseguir atendimento, o que no caso dos idosos ainda poderia ser agravado considerando-se fatores como capacidade funcional e disponibilidade da rede de apoio formal e informal.

Alguns idosos também não utilizaram um SAB porque não precisaram. Isso poderia estar vinculado à ideia de necessidade de procura do serviço apenas por motivo de doença, ou ainda em razão de o período de tempo investigado ter sido de seis meses.

Se, por um lado, alguns idosos que não utilizaram um SAB consideravam de melhor qualidade um serviço privado ou tiveram problemas de acesso, por outro, alguns usuários os consideravam um bom serviço e bem localizado. Essas respostas podem estar relacionadas tanto aos diferentes graus de satisfação dos idosos com os serviços da região como à diferenciação das ações de saúde oferecidas e de fatores relacionados ao acesso geográfico. Chamam a atenção ainda as respostas referentes à gratuidade dos serviços, quando, na verdade, esses serviços são pagos indiretamente por toda a população. No entanto, a gratuidade poderia representar que esses ido- 
sos utilizam um SAB por não terem condições de arcar com custos nos serviços privados, mas que, se tivessem possibilidade, os utilizariam.

Observa-se que a grande maioria dos idosos considerou a unidade de saúde que utilizava próxima à sua residência. Entretanto, alguns relataram utilizar uma unidade de saúde diferente daquela definida pela área de abrangência na política municipal, ou utilizar a sua unidade de referência além de outra, fornecendo outros endereços de moradia ou justificando que residiam em ruas limítrofes da área de abrangência. Apesar de não haver uma pergunta específica no instrumento sobre essa questão, os idosos referiram durante as entrevistas utilizar outra unidade por facilidade de deslocamento via transporte público, porque a considerava mais próxima de sua residência ou, ainda, por disponibilidade e qualidade de recursos humanos/materiais. Muitas vezes a unidade pode ser próxima à residência, mas na ocorrência de obstáculos tais como fortes aclives ou ruas movimentadas a serem atravessadas, o idoso prefere utilizar um transporte público que lhe permita descer em frente ao serviço de saúde, mesmo que isso exija maior tempo de deslocamento. Verifica-se ainda que esses achados vem ao encontro das observações de Venturi et al. ${ }^{20}$ e Ramos e Lima $^{24}$ de que a procura por atendimento nos SAB é influenciada pelo tipo de serviço ofertado e pelas experiências bem-sucedidas por parte dos usuários.

A maioria dos participantes do presente estudo que utilizava um SAB relatou deslocar-se a pé e de ônibus. Já o tempo para deslocamento até a unidade foi de 5 a 40 minutos. No estudo de Ramos e Lima ${ }^{24}$, foram citados pelos usuários os mesmos meios de deslocamento, e o tempo despendido até a unidade variou de 5 a 35 minutos, sendo semelhante ao referido na presente investigação, apesar de o estudo citado não ter sido realizado somente com idosos.

No que se refere à resolutividade, os serviços de atenção básica parecem estar atendendo grande parte das demandas dos idosos, pois a maioria deles considera os serviços como resolutivos. Em estudo para conhecer a percepção de resolutividade dos serviços de saúde pela população em geral, Turrini et al. ${ }^{11}$ identificaram que quase a metade dos entrevistados que referiram alguma morbidade nos 15 dias anteriores à entrevista e procuraram um serviço do Sistema Único de Saúde (SUS) percebeu-os como resolutivos. Mesmo considerando-se o período restrito investigado no estudo citado e que foram investigados serviços de todos os níveis de complexidade, é impor- tante salientar que apenas 14,8\% dos usuários do SUS perceberam não ter seu problema resolvido, sendo que os restantes ainda se encontravam em atendimento. Talvez, na presente investigação, as expectativas dos idosos em relação aos serviços que se ocupam da atenção básica ainda sejam pequenas.

O uso quase que exclusivo dos serviços de saúde públicos para consultas foi observado em estudo com dados da $\mathrm{PNAD}^{9}$, e as consultas médicas foram as mais procuradas em estudo realizado em SAB com idosos de Viçosa $(\mathrm{MG})^{20}$. Na presente investigação, consulta médica e imunização foram as mais citadas, sendo que a imunização deve ter apresentado maior proporção em razão de a presente investigação enfocar, especificamente, a finalidade de uso dos SAB e considerando a faixa etária específica deste estudo, para a qual a imunização para influenza é disponibilizada. Chama a atenção que poucos idosos relataram ter procurado o serviço para consulta com outros profissionais, demonstrando o enfoque mais centrado no atendimento médico.

A maioria dos idosos que utilizou um SAB o fez mais de uma vez em um período de seis meses. Os resultados parecem ser coerentes com maior utilização dos serviços de saúde por grupos etários extremos da população, ainda que na presente investigação tenha sido avaliada a frequência de utilização de um SAB, enquanto outros estudos avaliaram a procura por consultas médicas (mais de três por ano) em grupos populacionais semelhantes ${ }^{1,2,20}$.

O perfil sociodemográfico e as condições de saúde autorreferidas dos idosos que participaram da presente investigação foram analisados por Paskulin e Vianna ${ }^{25}$, verificando que $67,8 \%$ eram do sexo feminino, $84 \%$ encontravam-se na faixa de 60 aos 79 anos e $81 \%$ consideravam-se saudáveis.

Segundo a $\mathrm{PNAD}^{19}$, a utilização dos postos ou centros de saúde diminuía à medida que aumentava o rendimento familiar mensal. Considerando a classe econômica e escolaridade como proxys, também se observou na presente investigação uma redução na utilização dos SAB à medida que aumentava a escolaridade. Outras pesquisas também demonstraram que os idosos de classes econômicas mais privilegiadas ou de mais alta escolaridade utilizam mais os serviços privados ou planos de saúde, enquanto os de classes menos favorecidas dependiam exclusivamente do SUS $^{3,7,26-28}$. Associações entre utilização de serviços de saúde e condições econômicas também ocorrem em sistemas de saúde de países desen- 
volvidos, mas com características diversas. Estudo realizado em 21 países não encontrou evidências de iniquidade de acesso a atendimentos com médicos gerais comunitários; porém, identificou que pessoas de classes econômicas mais privilegiadas tinham mais acesso a consultas com especi$\operatorname{alistas}^{29}$. Entre idosos coreanos também foi identificada associação entre melhor posição socioeconômica e utilização de serviços preventivos ${ }^{30}$.

O fato de se perceber doente e de relatar ser portador de dano crônico também esteve associado a uma maior utilização de um SAB. Estudos no contexto internacional e nacional já demonstraram que as necessidades de saúde são inversamente proporcionais à posição socioeconômica $^{19,31,32}$ e que pior avaliação de saúde ou morbidade autorreferida estão associadas a maior utilização dos serviços de saúde em geral ou do SUS, tanto para idosos ${ }^{7,8,33}$ como para a população em geral $^{2,9,34}$.

Os resultados da presente investigação referentes à associação entre utilização do SAB com escolaridade e com percepção de saúde confirmam os achados de outros estudos e reforçam a influência dos determinantes sociais na saúde e no acesso aos serviços pela população idosa. Reforçam ainda a ideia de que atenção à saúde não envolve apenas melhorias no sistema de saúde, mas nas condições de vida como um todo.

No presente estudo, sexo não esteve associado à utilização dos SAB. No entanto, ao analisar a utilização dos serviços de saúde por idosos, Pinheiro e Travassos ${ }^{8}$ encontraram associação entre maior consumo dos serviços de saúde e idosas. Bós e Bós ${ }^{7}$, analisando os determinantes na escolha entre atendimento de saúde privado e público por idosos, identificaram que as idosas eram as que mais utilizavam os serviços privados. Travassos et al. ${ }^{35}$, com base em dados da PNAD de 1998, identificaram que as taxas de utilização dos serviços de saúde foram maiores para as mulheres, tanto nos casos de pessoas com restrição de atividades rotineiras por motivos de saúde quanto nos daquelas sem essa condição. Salienta-se, entretanto, que esses três estudos não enfocaram os $\mathrm{SAB}$, e que o último não abrange apenas pessoas idosas.

Também não foi encontrada associação entre grupo etário e a utilização dos SAB. Outros estudos que analisam a associação entre grupos etários e utilização dos serviços de saúde obtiveram resultados variados, talvez em razão de diferentes agrupamentos dos serviços de saúde utilizados. Bós e Bós ${ }^{7}$ identificaram estar a idade associada significativamente à utilização da rede privada, sendo que os idosos mais velhos utilizavam mais os serviços de saúde privados do que os idosos mais jovens. Já Pinheiro e Travassos ${ }^{8} \mathrm{e}$ Lima-Costa et al. ${ }^{1}$ não encontraram associação entre grupos etários e chances de consumo dos serviços ou de procura de atendimentos médicos, de modo geral, por pessoas idosas.

As variáveis estado conjugal, percepção de apoio social informal, arranjo domiciliar e AVD também não apresentaram associação com a utilização dos SAB. Outros estudos encontraram associação entre variáveis relacionadas à rede de apoio informal e utilização dos serviços de saúde ou de consultas médicas, seja para idosos, seja para a população adulta ${ }^{2,7,36}$. Talvez em razão de o presente estudo estar investigando apenas os $\mathrm{SAB}$, essas variáveis não tenham mostrado associação, permanecendo significativas aquelas com maior impacto. Em relação à capacidade funcional, é possível que a pequena proporção de idosos com dependência severa também tenha influenciado sua não associação com a utilização de um SAB.

\section{Conclusões}

Os resultados mostraram que os SAB foram utilizados por $49,7 \%$ dos idosos, motivados por sua localização, qualidade e "gratuidade". O principal motivo relatado para a não utilização de um SAB foi possuir plano de saúde. A grande maioria dos idosos considerou o $\mathrm{SAB}$ próximo à sua residência, mas muitos idosos utilizavam unidades de saúde diferentes das definidas na política municipal. As principais finalidades de uso dos SAB foram vacinação, consultas médicas e busca por medicação. Os fatores associados negativamente à utilização do SAB foram "escolaridade" e o fato de "perceber-se saudável", e a variável associada positivamente foi "autorrelato de dano crônico".

Ainda que mudanças na estrutura do sistema de saúde estejam sendo perseguidas desde a implantação do SUS, observou-se que as ações de saúde buscadas pelos idosos e as próprias demandas e expectativas destes parecem centrarse no atendimento individual, prestado pelo profissional médico e com enfoque curativo. Verifica-se ainda que os idosos expostos a situações de fragilidade relacionadas a condição econômica, percepção subjetiva de doença e que relatavam serem portadores de dano crônico foram os que mais utilizaram um SAB. De certo modo, esses resultados podem estar demonstrando sinais de 
equidade do sistema, ainda que as ações de saúde disponibilizadas aos idosos sejam limitadas.

A influência dos aspectos econômicos na utilização e no acesso aos serviços, identificada nesta investigação, tem sido bastante divulgada na literatura, como já discutido anteriormente. Para além do âmbito econômico, este estudo remete a questões importantes que podem nortear o planejamento local da atenção básica ao idoso, assim como reforça a necessidade de implantação do princípio de organização da atenção básica ao idoso em dois eixos: o dos idosos independentes e o dos idosos frágeis ${ }^{21}$. Desse modo, as necessidades específicas de cada grupo podem ser contempladas. Para atender a esse princípio, as alternativas passam necessariamente pelo estabelecimento de estratégias de promoção da saúde, de prevenção de danos crônicos e de suas complicações, além de ações de reabilitação. En- volve ainda alterar as representações presentes em nossa sociedade relacionadas às questões sobre o processo saúde-doença, ser saudável e o papel dos serviços de saúde, tanto na perspectiva dos usuários como na dos profissionais envolvidos com a assistência e formulação de políticas para esse grupo populacional. A ampliação do acesso, a partir dos princípios do SUS, é uma questão que tem sido bastante explorada no contexto nacional, tendo em vista um sistema de saúde que prevê atendimento a todos, mas que não dispõe de recursos para isso. No entanto, se o impacto do envelhecimento populacional e o consequente aumento da utilização dos serviços pelos idosos não forem pensados, a racionalização dos recursos no contexto atual trará importantes consequências na qualidade de vida dos atuais e futuros idosos e, também, nos próprios gastos do sistema de saúde.

\section{Colaboradores}

LMG Paskulin elaborou o projeto, realizou a coleta e análise dos dados e redigiu o artigo; DB Valer colaborou na análise dos dados e na redação; LAC Vianna participou da concepção do projeto, da análise dos dados e da revisão crítica do artigo.

\section{Referências}

1. Lima-Costa FL, Barreto S, Giatti L. Condições de saúde, capacidade funcional, uso de serviços de saúde e gastos com medicamentos da população idosa brasileira: um estudo descritivo baseado na Pesquisa Nacional por Amostra de Domicílios. Cad Saude Publica 2003; 19(3):735-743.

2. Mendonza-Sassi R, Béria J. Utilización del serviços de salud: una revisión sistematica sobre los fatores associados. Cad Saude Publica 2001; 17(4):819-832.

3. Veras R, Parahyba MI. O anacronismo dos modelos assistenciais para os idosos na área da saúde: desafios para o setor privado. Cad Saude Publica 2007; 23(10):2479-2489.

4. Travassos C, Martins M. Uma revisão sobre os conceitos de acesso e utilização de serviços de saúde. Cad Saude Publica 2004; 20(Supl.2):190-198.

5. Unglert C. Territorialização em sistemas de saúde. In: Mendes E, organizador. Distrito sanitário:o processo social de mudança das práticas sanitárias do Sistema Único de Saúde. 4a ed. São Paulo, Hucitec; Rio de Janeiro: Abrasco; 1999. p. 221-236.

6. Cosenza G. Distribuição espacial e acesso da população aos serviços de saúde. In: Pierantoni C, Vianna M, organizadores. Gestão de sistemas de saúde. Rio de Janeiro: UERJ; 2003. p. 133-168.

7. Bós A, Bós A. Determinantes na escolha entre atendimento de saúde privada e pública por idosos. Rev Saude Publica 2004; 38(1):113-120.

8. Pinheiro RS, Travassos C. Estudo da desigualdade na utilização de serviços de saúde por idosos em três regiões da cidade do Rio de Janeiro. Cad Saude Publica 1999; 15(3):487-496. 
9. Ribeiro MCSA, Barata RB, Almeida MF, Silva ZP. Perfil sociodemográfico e padrão de utilização de serviços de saúde para usuários e não-usuários do SUS PNAD 2003. Cien Saude Colet 2006; 11(4):1011-1022.

10. Travassos C, Viacava F. Acesso e uso de serviços de saúde em idosos residentes em áreas rurais, Brasil, 1998 e 2003. Cad Saude Publica 2007; 23(10):2490-2502.

11. Turrini RNT, Lebrão ML, Cesar CLG. Resolutividade dos serviços de saúde por inquérito domiciliar: percepção do usuário. Cad Saude Publica 2008; 24(3):663-674.

12. Starfield B. Atenção Primária: equilíbrio entre necessidades de saúde, serviços e tecnologia. Brasília: Unesco, Ministério da Saúde; 2002.

13. Piccini RX, Fachini LA, Tomasi E, Thumé E, Silveira DS, Siqueira FV, Rodrigues MA. Necessidade de saúde comuns aos idosos: efetividade na oferta e utilização em atenção básica à saúde. Cien Saude Colet 2006; 11(3):657-667.

14. Haggerty JL, Pineault R, Beaulieu MD, Brunelle Y, Gauthier J, Goulet F, Rodrigue J. Practice features associated with patient-reported acessibility, continuity, and coordination of Primary Health Care. Ann Fam Med 2008; 6(2):116-123.

15. Almeida Filho N, Rouquayrol Z. Elementos de metodologia epidemiológica. In: Almeida Filho N, Rouquayrol Z. Epidemiologia e saúde. $6^{\text {a }}$ ed. Rio de Janeiro: Medsi; 2003. p. 149-178.

16. Kirkwood B, Stern NJ. Essential medical statistics. $2^{\text {a }}$ ed. Massachusetts: Blackwell; 2003.

17. Ramos LR, Rosa TEC, Oliveira ZM, Medina MCG, Santos FRG. Perfil do idoso em área metropolitana na Região Sudeste do Brasil: resultados de inquérito domiciliar. Rev Saude Publica 1993; 27(2):87-94.

18. Idler EL, Benyamini Y. Self-rated health and mortality: a review of twenty-seven community studies. J Health Soc Behav 1997; 38(1):21-37.

19. Fundação Instituto Brasileiro de Geografia e Estatística. Pesquisa Nacional por Amostra de Domicílios 2003: acesso e utilização de serviços de saúde. Brasília: IBGE; 2003. [acessado 2008 abr 15]. Disponível em: http:// www.ibge.gov.br/home/estatistica/populacao/ trabalhoerendimento/pnad2003/saude/saude2003.pdf

20. Venturi I, Rosado LEFP, Cotta RMM, Rosado GP, Doimo LA, Tinoco ALA, Ribeiro, RCL. Identificação da área de influencia do serviço de atenção básica do sistema público de saúde à população idosa, município de Viçosa-MG. Cien Saude Colet 2008; 13(4):1293-1204.

21. Brasil. Portaria no 2.528, de 19 de outubro de 2006. Aprova a Política Nacional de Saúde da Pessoa Idosa. Brasília: Ministério da Saúde; 2006. [acessado 2008 fev 26]. Disponível em: http://portal.saude.gov.br/ portal/arquivos/pdf/2528_pnspi.pdf

22. Travassos C. Fórum: equity in access to health care. Cad Saude Publica 2008; 24(5):1159-1161.

23. Travassos C, Oliveira EXG, Viacava F. Desigualdades geográficas e sociais no acesso aos serviços de saúde no Brasil: 1998 e 2003. Cien Saude Colet 2006; 11(4):975-986.
24. Ramos DD, Lima MADS. Acesso e acolhimento aos usuários em uma unidade de saúde de Porto Alegre, Rio Grande do Sul, Brasil. Cad Saude Publica 2003; 19(1):27-34.

25. Paskulin LMG, Vianna LAC. Perfil sociodemográfico e condições de saúde auto-referidas de idosos de Porto Alegre. Rev Saude Publica 2007; 41(5):757-768.

26. Cesar C, Paschoal S. Uso dos serviços de saúde. In: Lebrão ML, Duarte Y, organizadores. SABE-Saúde, bem-estar e envelhecimento: o projeto SABE no município de São Paulo: uma abordagem inicial. Brasília: Organização Pan-Americana da Saúde; 2003. p. 225-238.

27. Noronha KVMS, Andrade MV. Desigualdades sociais em saúde e na utilização dos serviços de saúde entre os idosos na América Latina. Rev Panam Salud Publica 2005; 17(5/6):410-418.

28. Wallace S, Gutiérrez V. Equity of access to health care for older adults. Rev Panam Salud Publica 2005; 17(5/6):394-409.

29. Van Doorslaer E, Masseria C, Koolman X. Inequalities in access to medical care by income in developed countries. Can Med Assoc J. 2006; 174(2):177-183.

30. Chum H, Kim IH. Socioeconomic inequalities in preventive services among the elderly: results from medical checkup, cancer check, and BP check. $J$ Prev Med Public Health 2007; 40(5):404-410.

31. Evans RG, Barer ML, Marmot TR, editors. Why are some people healthy and others not? New York: Aldine de Gryer; 1994.

32. Neri MC, Soares WL. Estimando o impacto da renda na saúde através de programas de transferência de renda aos idosos de baixa renda no Brasil. Cad Saude Publica 2007; 23(8):1845-1856.

33. Lima-Costa F, Firmo JOA, Uchoa E. The structure of self-rated health among older adults: the Bambuí health and ageing study. Rev Saude Publica 2004; 38(6):827-834.

34. Almeida MF, Barata RB, Monteiro CV, Silva ZP. Prevalência de doenças crônicas auto-referidas e utilização de serviços de saúde, PNAD/1998, Brasil. Cien Saude Colet 2002; 7(4):743-756.

35. Travassos C, Viacava F, Pinheiro R, Brito A. Utilização dos serviços de saúde no Brasil: gênero, características familiares e condição social. $\mathrm{Rev} P a$ nam Salud Publica 2002; 11(5):365-373.

36. Capilheira MF, Santos IS. Fatores individuais associados à utilização de consultas médicas por adultos. Rev Saude Publica 2006; 40(3):436-443.

Artigo apresentado em 29/05/2008

Aprovado em 18/09/2008

Versão final apresentada em 01/06/2009 